\title{
Neutrophil Induced Oxidative Injury of Cardiac Myocytes A Compartmented System Requiring CD11b/CD18-ICAM-1 Adherence
}

\author{
Mark L. Entman, ${ }^{*}$ Keith Youker, ${ }^{*}$ Takachika Shoji, * Gilbert Kukielka, * Scott B. Shappell, ${ }^{*}$ \\ Addison A. Taylor, ${ }^{\star}$ and C. Wayne Smith ${ }^{* \$}$ \\ * Section of Cardiovascular Sciences, The Methodist Hospital and The DeBakey Heart Center, Department of Medicine, Baylor College \\ of Medicine; ${ }^{\ddagger}$ Speros P. Martel Laboratory of Leukocyte Biology, Department of Pediatrics, Texas Children’s Hospital; and \\ ${ }^{\S}$ Department of Microbiology and Immunology, Baylor College of Medicine, Houston, Texas 77030
}

\begin{abstract}
We have previously shown that cytokines and postischemic cardiac lymph induce expression of intercellular adhesion molecule-1 (ICAM-1, CD54) on canine adult cardiac myocytes. ICAM-1 expression allows adherence of activated neutrophils to myocytes that is blocked by anti-CD18 $\mathrm{mAb}, \mathrm{R15.7}$, or antiICAM-1 mAb, CL18/6. Interleukin 1, tumor necrosis factor$\alpha$, or interleukin 6-stimulated cardiac myocytes were loaded with $2^{\prime}, 7^{\prime}$-dichlorofluorescin, and oxidation to the fluorescent dichlorofluorescein was monitored. Fluorescence and neutrophil/myocyte adherence followed the same time course, and both were blocked by monoclonal antibodies to CD18, CD11b, and ICAM-1, but mAb R7.1, recognizing a functional epitope on CD11a, was not inhibitory. The iron chelator, desferroxamine, and the hydroxyl radical scavenger, dimethylthiourea, did not inhibit neutrophil adherence, but completely inhibited fluorescence. In contrast, the extracellular oxygen radical scavengers superoxide dismutase and catalase, and the extracellular iron chelator, starch-immobilized desferroxamine, did not affect either fluorescence or adherence. Under the experimental conditions used, no superoxide production could be detected in the extracellular medium. Fluorescence microscopy demonstrated that fluorescence began within $5 \mathrm{~min}$ after neutrophil adherence to an individual myocyte, and myocyte contracture followed rapidly. Fluorescent intensity was highest initially at the site of myocyte-neutrophil adherence. When only neutrophils were loaded with $2^{\prime}, 7^{\prime}$-dichlorofluorescein, fluorescence was observed only in those neutrophils adhering to the cardiac myocytes. Thus, adherence dependent on Mac-1 (CD11b/ CD18) and ICAM-1 (CD54) activates the neutrophil respiratory burst resulting in a highly compartmented iron-dependent myocyte oxidative injury. (J. Clin. Invest. 1992. 90:13351345.) Key words: adhesion-dependent oxidative burst $\bullet$ Mac1-ICAM-1 adhesion - neutrophil-induced myocyte injury • compartmented myocyte oxidative injury
\end{abstract}

\section{Introduction}

Reperfusion of ischemic myocardium promotes influx of neutrophils which is most rapid within the first $2 \mathrm{~h}$ after reflow

Address correspondence to Mark L. Entman, M.D., Department of Medicine, Cardiovascular Sciences, Baylor College of Medicine, One Baylor Plaza, Houston, TX 77030.

Received for publication 2 March 1992 and in revised form 1 May 1992.

J. Clin. Invest.

(c) The American Society for Clinical Investigation, Inc.

$0021-9738 / 92 / 10 / 1335 / 11 \$ 2.00$

Volume 90, October 1992, 1335-1345 begins ( 1 ) and may continue at a low level for at least $48 \mathrm{~h}$ (2). The peak accumulation of these cells occurs between 24 and 48 $h$ (3). While evidence derived from experimental animal models indicates that the accumulated neutrophils injure the myocardium (2), the specific mechanisms affecting this injury have not been defined. Two general mechanisms have been studied. The first arises from the observations that neutrophils can lodge in small blood vessels and thereby obstruct local blood flow (4-6). The second arises from the concept that neutrophil emigration brings these cells in close proximity to myocytes where secretory products would have enhanced injurious effects, particularly if the neutrophils adhered to the myocytes. In previous studies, we examined the mechanisms of neutrophil adhesion to cardiac myocytes in vitro (7), and found that intercellular adhesion occurred if the myocytes were stimulated to express intercellular adhesion molecule-1 (ICAM-1, ' CD54) (8), and the neutrophils were stimulated to enhance CD18-dependent adhesion (7). Stimulation of myocytes with cytokines such as IL-1, tumor necrosis factor- $\alpha$ (TNF $\alpha$ ) and IL-6 or by postischemic cardiac lymph induced expression of ICAM-1 ( 8, 9), and stimulation of neutrophils with zymosan activated serum (a source of C5a) or platelet-activating factor (PAF) promoted $\beta 2$ integrin-dependent adhesion that was blockable by anti-CD18 and anti-ICAM-1 (mAbs) $(7,8)$. Adhering neutrophils were apparently cytotoxic, as indicated by the sustained contraction often observed in myocytes after neutrophil adhesion (7). In the present report, we address the potential pathogenic role of this intercellular adhesion by assessing possible mechanisms of cytotoxicity. Two experimental approaches were taken. (a) Adhesion was studied in vitro as previously reported, and the contribution of specific adhesion molecules was investigated using appropriate mAbs. $(b)$ Myocytes were loaded with $2^{\prime}, 7^{\prime}$-dichlorofluorescein (DCFH), a probe used to indicate intracellular oxidation after neutrophil adhesion.

\section{Methods}

Isolation of canine cardiac myocytes. Healthy mongrel dogs weighing $10-15 \mathrm{~kg}$ were anesthetized using sodium pentobarbital. The heart was removed through the left lateral chest under sterile conditions, and immediately placed in ice cold saline. The aorta was then cannulated using a tubing adapter suitable for the individual heart, and the procedure for obtaining isolated myocytes was followed exactly as described previously (7). Preparations with a viability of $>80 \%$ were used in incubation experiments with neutrophils. Cells were then placed on ice and used within one to two days. Viability was not appreciably altered over this period.

1. Abbreviations used in this paper: CVJEC, canine jugular vein endothelial cells; DCFH, 2',7'-dichlorofluorescein; ICAM-1, intercellular adhesion molecule-1; PAF, platelet-activating factor; TNF- $\alpha$, tumor necrosis factor- $\alpha$; ZAS, zymosan-activated serum. 
Neutrophil isolation. Canine neutrophils were isolated from citrate anticoagulated venous blood using techniques previously described for the isolation of human neutrophils $(10,11)$. This yielded a preparation of cells $>95 \%$ neutrophils with $>99 \%$ viability. These cells were suspended in Dulbecco's PBS and stored at $4^{\circ} \mathrm{C}$ for up to $4 \mathrm{~h}$.

Monoclonal antibodies. Anti-CD18 mAb R15.7 (IgG1) (7) and anti-CD1 la mAb R7.1 (IgG1) $(12,13)$ were provided by Dr. R. Rothlein (Boehringer Ingelheim Pharmaceuticals Inc., Ridgefield, CT). Anti-CD1 $1 \mathrm{~b}$ mAb was isolated from a hybridoma clone (accesson No. HB-9510) obtained from American Type Culture Collection (ATCC, Rockville, MD). This mAb was designated MY904 and defined as anti-human CD11b. The preparations of purified IgG used in the current studies were isolated from ascites fluid as previously described (8). M1 / 70 (anti-CD1 1b, IgG2b) was obtained from ATCC and isolated from culture supernatant as previously described (14). Two control mAbs, SG10G8 (IgG1) and SG2G8 (IgG1 ) against canine neutrophils, were prepared as previously described (8). These antibodies bound to neutrophils at about the same level as R15.7, but did not bind to canine endothelial cells or myocytes. They served as binding controls in adhesion assays and have not been found to inhibit adhesion. Monoclonal antibodies IgG1 (Damon Biotech, Needham Heights, MA) and 4A5 (IgG1, prepared as previously described ) (10) without binding specificity for canine cells served as nonbinding controls. Anticanine ICAM-1 mAbs CL18/6 and CL18/1D8 were prepared as previously described (8). In some instances, mAbs were used as $F(a b)_{2}^{\prime}$ fragments, prepared as previously described (15).

Indirect immunofluorescence assessments of the surface binding sites for monoclonal antibodies were performed using saturating concentrations of the monoclonal antibodies and FITC-conjugated goat anti-mouse IgG (16). Analysis was carried out with a FACScan ${ }^{\otimes}$ (Becton Dickinson, Mountain View, CA) flow cytometer. The nonbinding IgG1 mAbs served as control.

Canine neutrophil adherence to canine endothelial monolayers. Canine jugular vein endothelial cells (CJVEC) were obtained by a modification of the method of Ford (17) as previously described (7). Coverslips with attached endothelial monolayers were inserted in adhesion chambers, and adherence and transendothelial migration of isolated canine neutrophils was determined in the absence of shear stress using a visual assay as previously described $(10,18)$. In experiments with stimulated endothelial cells, monolayers were exposed to cytokines for $3 \mathrm{~h}$ at $37^{\circ} \mathrm{C}$, then rinsed by dipping the coverslip five times in two changes of PBS before being inserted into the adherence chambers. In experiments with monoclonal antibodies, neutrophils were exposed to the antibodies at room temperature for $5 \mathrm{~min}$ before the cell suspension containing the antibodies was injected into the adherence chambers.

Canine neutrophil-myocyte adherence. Isolated canine myocytes were suspended in isolation medium at a concentration of $50,000 / \mathrm{ml}$ as previously described (7). Neutrophils and myocytes were coincubated in a vol of $0.4 \mathrm{ml}$ at a neutrophil to myocyte ratio of $50: 1$, for various times up to $60 \mathrm{~min}$ at $37^{\circ} \mathrm{C}$. The cells were resuspended and small aliquots transferred to microscope slides for examination under phase contrast or differential interference contrast optics. For each preparation, the number of neutrophils adherent to each of $\mathbf{2 0 0}$ myocytes was counted. Samples were coded so that data collection was performed without knowledge of the specific experimental conditions. Myocytes were incubated in the presence or absence of cytokines including human recombinant IL-1 $\beta$ (Genzyme Corp., Boston, MA), human recombinant IL-6 (Genzyme Corp.), or human recombinant TNF $\alpha$ (Boehringer-Ingelheim), for various times at $37^{\circ} \mathrm{C}$ before addition of the neutrophils. In experiments with stimulated neutrophils, zymosan-activated serum (ZAS, prepared as previously described) (11) was added immediately before the neutrophil suspension was mixed with the suspension of myocytes. mAb were added to the neutrophil-myocyte suspension at the beginning of incubation, and were present throughout the incubation period.

Adherence-dependent oxidation. Isolated myocytes were preincubated for $3 \mathrm{~h}$ with interleukin $1(4 \mathrm{U} / \mathrm{ml})$ as described above. Subsequently, they were loaded with $2^{\prime}, 7^{\prime}$ dichlorodihydrofluoroscein diace- tate (DCFH; Molecular Probes, Inc., Eugene, OR) by incubating with a concentration of $100 \mu \mathrm{M}$ for $20 \mathrm{~min}$ at $37^{\circ} \mathrm{C}$, washed twice, and suspended with neutrophils under identical circumstances to the protocol used for adherence (i.e., ZAS was added as a chemotactic stimulus to provoke neutrophil adhesion). Control experiments in the absence of either cytokine or ZAS were also performed. Three assessments were made: ( $a$ ) Fluorometric studies were performed by monitoring cellular fluorescence in a fluorometer (Perkin-Elmer Cetus Instruments, Norwalk, CT; excitation $488 \mathrm{~nm}$ and emission of $521 \mathrm{~nm}$ ) after ZAS addition. This incubation was carried out at $37^{\circ} \mathrm{C}$. (b) Direct observation of individual cells under fluorescence microscopy was video recorded and evaluated using Optimas image analysis software (Bioscan Inc., Edmonds, WA). (c) Superoxide release into the culture medium was measured using cytochrome $c$ as previously described (19). The superoxide generating system, xanthine and xanthine oxidase, was used as a defined extracellular source of reactive oxygen as previously described (19).

Reagents. Desferroxamine, superoxide dismutase (SOD), catalase, xanthine, xanthine oxidase, phorbol myristate acetate, (PMA) endotoxin (LPS), PAF, and dimethylthiourea were obtained from Sigma Chemical Co. (St. Louis, MO); the starch immobilized desferroxamine was a gift of Dr. B. Hedlund (Biomedical Frontiers, Inc., Minneapolis, MN).

\section{Results}

Neutrophil/endothelial interactions. An important focus of the current study was the evaluation of $\beta 2$ integrin-dependent adhesion of canine neutrophils to isolated canine cardiac myocytes, and the approach to distinguishing the relative contributions of CD1 1a/CD18 (LFA-1) and CD11b/CD18 (Mac-1) was the use of subunit specific mAbs. The anti-CD1la and anti-CD11b mAbs used in the current study are known to bind to human $\beta 2$ integrin subunits and to block their specific functions $(10,15)$. However, the ability of these mAbs to block the adhesive functions of canine $\beta 2$ integrins has not been previously documented. Such cross-species reactivity has been published for the anti-CD18 MAb, R15.7 (7, 15). R7.1 (anti-

Table I. Binding of MAbs R7.1 and MY904 to Human and Canine Leukocytes

\begin{tabular}{|c|c|c|c|c|}
\hline \multirow[b]{2}{*}{ Cell type } & \multicolumn{2}{|c|}{ R7.1 } & \multicolumn{2}{|c|}{ MY904 } \\
\hline & Canine & Human & Canine & Human \\
\hline Neutrophils & $156(98)^{*}$ & $134(99)$ & $57(99)$ & $99(100)$ \\
\hline \multicolumn{5}{|l|}{ Stimulated $^{\ddagger}$} \\
\hline neutrophils & $145(98)$ & $196(99)$ & $344(98)$ & $952(100)$ \\
\hline Lymphocytes & $211(90)$ & $272(97)$ & $104(24)$ & $84(34)$ \\
\hline Monocytes & $231(92)$ & $295(96)$ & $83(94)$ & $100(94)$ \\
\hline $\begin{array}{l}\text { Stimulated } \\
\text { monocytes }\end{array}$ & $234(94)$ & $321(96)$ & $355(61)^{8}$ & $1090(54)^{8}$ \\
\hline
\end{tabular}

* Binding of these mAbs to leukocytes in whole blood samples was evaluated using flow cytometry. The cell type was identified by forward and side scatter characteristics, and the mean fluorescence channel and percent positive cells (value in parentheses) were determined for each cell type and $\mathrm{mAb}$. Cells were considered positive for the specific mAb if the level of fluorescence exceeded that of cells exposed to nonbinding, isotype-matched antibodies. ${ }^{\ddagger}$ Human neutrophils and monocytes were stimulated by the addition of $10 \mathrm{nM}$ fMLP to the whole blood sample for $15 \mathrm{~min}, 37^{\circ} \mathrm{C}$, before preparing the cells for flow cytometry. Canine neutrophils and monocytes were stimulated by the addition of $1 \% \mathrm{ZAS}$ for $15 \mathrm{~min}, 37^{\circ} \mathrm{C}$. The value in parentheses indicate the percent monocytes with MY904 binding beyond that of $97 \%$ unstimulated monocytes. 


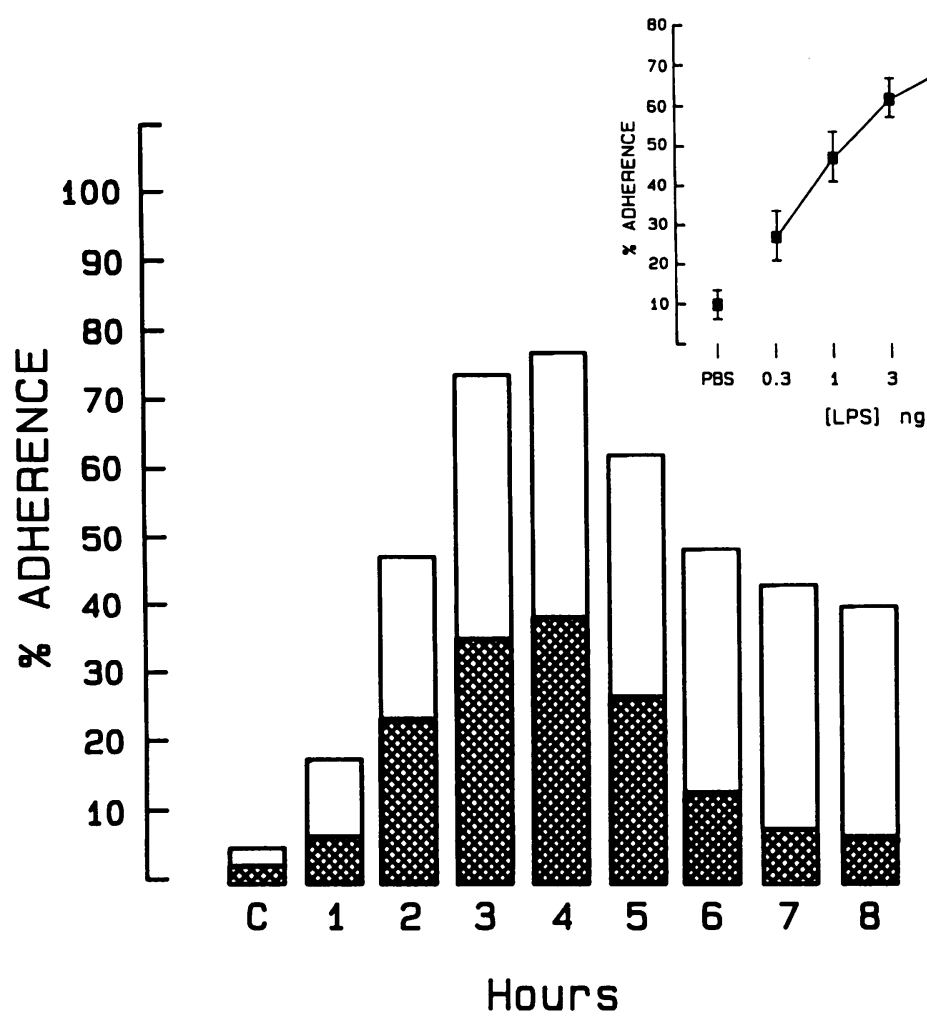

Figure 1. Stimulation of CJVEC adhesiveness for canine neutrophils by LPS. The inset graph demonstrates the effects of incubating CJVEC monolayers for $4 \mathrm{~h}$ in different concentrations of LPS on adhesion of isolated and previously unstimulated canine neutrophils. Adhesion was determined under static conditions after allowing neutrophils to contact the monolayers for $500 \mathrm{~s}$. Based on this dose-response study $(n=4), 10 \mathrm{ng} / \mathrm{ml}$ was selected for the time course shown where endothelial monolayers were incubated with LPS for the time indicated. The effect of anti-CD18 mAb, R15.7, is also shown with the level of adhesion indicated by the height of the cross-hatched bars, and the adhesion without addition of the mAb is indicated by the height of the open bars ( $n$ $=3,1$ SD did not exceed $20 \%$ of the mean).

CD11a) and MY904 (anti-CD11b) were both evaluated for their ability to distinguish LFA-1 and Mac-1 dependent adhesion of canine neutrophils. As shown in Table I, R7.1 exhibited the binding characteristics on canine leukocytes of an antiCD1 1a in that it bound to almost all leukocytes and there was little change in the binding to neutrophils or monocytes after chemotactic stimulation. MY904 exhibited a pattern of binding distinct from R7.1, and the patterns seen with canine and human cells were quite similar and consistent with antiCD11b. Binding of MY904 to neutrophils and a portion of monocytes was markedly upregulated after stimulation with a chemotactic factor, and this mAb bound to only a portion of blood lymphocytes.

Canine neutrophil-endothelial interactions. CJVEC monolayers stimulated with LPS were found to become most adhesive for isolated and previously unstimulated canine neutrophils within 3-4 h (Fig. 1). The CD18-independent component of this adhesion (i.e., that adhesion not blocked by mAb R15.7, anti-CD18, shown as hatched bars in Fig. 1) peaked at 4 $\mathrm{h}$ and returned to low levels within $8 \mathrm{~h}$ of LPS stimulation, while the CD18-dependent component (indicated by the open portions of the bars in Fig. 1) peaked at $4 \mathrm{~h}$ and remained high throughout an 8-h observation (Fig. 1). The ability of $\mathrm{mAb}$ R7.1 (anti-CD11a) to reduce adhesion was evaluated at 4 and $18 \mathrm{~h}$ after LPS stimulation of CJVEC monolayers (Fig. 2), and this $\mathrm{mAb}$ was found to be as effective as anti-ICAM-1 (mAb CL18/6) and anti-CD18 (mAb R15.7). The anti-CD1 lb was not inhibitory in this experimental setting. Previous studies using anti-CD1 $1 \mathrm{a}$ and anti-CD1 $1 \mathrm{~b}$ mAbs with human neutrophil-endothelial cell adhesion gave essentially the same results (10). In contrast to this effect, anti-CD1 la mAbs have not been found to inhibit adhesion of chemotactically stimulated neutrophils to protein-coated glass $(15,20,21)$. This was found to be true for mAb R7.1 (Fig. 2). This mAb was not effective in reducing adhesion of PAF-stimulated canine neutrophils to keyhole limpet hemocyanin-coated glass, though this latter adhesion was blocked by anti-CD1 1b (MY904) and anti-CD18 (R15.7) mAbs.
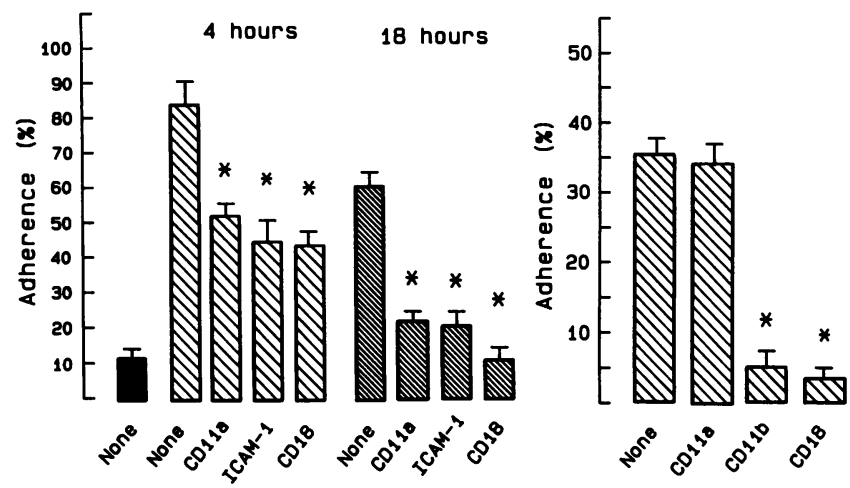

Figure 2. Effects of anti-CD11a and anti-CD11b mAbs on canine neutrophil adhesion. Results from three distinct experimental conditions are shown. In the graph on the left CJVEC monolayers were stimulated with LPS $(10 \mathrm{ng} / \mathrm{ml})$ for $4 \mathrm{~h}$ or $18 \mathrm{~h}$, rinsed in PBS, and placed in static adhesion chambers. When mAbs were present, they were incubated with the appropriate cells $15 \mathrm{~min}$ before the adhesion assay and retained with the cells throughout the period of time allowed for adhesion. The solid bar indicates the level of adhesion when neither monolayers nor neutrophils were stimulated. None indicates that $\mathrm{mAbs}$ were not added. The graph at the right presents results of canine neutrophil adhesion to keyhole limpet hemocyanin-coated glass under static conditions after a contact time of $500 \mathrm{~s}$. Neutrophils were preincubated with $\mathrm{mAb}$ for $15 \mathrm{~min}$ before the adhesion assay. $\mathrm{mAbs}$ used in these conditions recognize the antigens listed below the bars: CD1 la, R7.1 (10 $\mu \mathrm{g} / \mathrm{ml}, \mathrm{IgG})$; CD1 lb, MY904 (10 $\mu \mathrm{g} / \mathrm{ml}$, IgG); CD18, R15.7 (10 $\mu \mathrm{g} / \mathrm{ml}, \mathrm{IgG})$; ICAM-1, CL18/6 (20 $\mu \mathrm{g} / \mathrm{ml}$, $\left.\mathrm{F}(\mathrm{ab})_{2}^{\prime}\right) .{ }^{*} P<0.01, n=4$. 
Studies with human cells have shown that the adhesion of previously unstimulated neutrophils to cytokine-stimulated endothelial cells is partially CD18/ICAM-1 dependent, and the principal $\beta 2$ integrin involved in this adhesion is LFA-1 (10, 22 ). Additionally, chemotactic stimulation of neutrophils enhances ICAM-1-dependent adhesion to endothelial cells and recruits the participation of Mac-1 (10, 23, 24). Such experiments were repeated using canine cells (Fig. 3) with results similar to those obtained with human cells. mAb R7.1 (antiCD1 1a), but not MY904 (anti-CD1 1b) inhibited the adhesion of previously unstimulated canine neutrophils to rTNF $\alpha$ stimulated CJVEC. After stimulation of canine neutrophils with exogenous PAF, adhesion to rTNF $\alpha$-stimulated CJVEC was enhanced, and both R7.1 and MY904 significantly inhibited this adhesion. When used together, the inhibitory effects of R7.1 and MY904 were almost additive, indicating that this experimental condition leads to a cooperative function of LFA- 1 and Mac-1 in the adhesion of chemotactically stimulated canine neutrophils to cytokine-stimulated CJVEC.

Transendothelial migration of canine neutrophils. After adhering, both human and canine neutrophils rapidly migrate beneath cytokine-stimulated endothelial cell monolayers (18, 25 ). In both species, this behavior is almost completely inhibited by anti-CD18 mAbs (18, 25), and LFA-1 and Mac-1 have been shown to cooperate in the migration of human cells (10, 25). mAbs R7.1 (anti-CD1 1a) and MY904 (anti-CD1 1b) each partially inhibited transendothelial migration of canine neutrophils through LPS-stimulated CJVEC monolayers, and when combined, resulted in inhibition that was statistically equivalent to that produced by anti-CD18 mAb R15.7 and antiICAM-1 mAb CL18/6 (Fig. 4).

Neutrophil/cardiac myocyte interactions. The results presented above distinguish mAbs R7.1 (anti-CD11a) and MY904 (anti-CD11b) functionally. R7.1 inhibits neutrophil functions known to be carried out by $\operatorname{LFA}-1(10,22,25,26)$, and MY904 inhibits neutrophil functions known to be carried out by Mac-1 (10, 22, 25, 26). When used in combination, these two mAbs appear functionally equivalent to an antiCD18. Thus, it was possible to address the question of the relative contributions of LFA-1 and Mac-1 to neutrophil/myocyte interactions.

Neutrophil adhesion and myocyte intracellular oxidation. Direct observations of individual IL-1-stimulated myocytes loaded with DCFH (Fig. 5) and incubated for up to $1 \mathrm{~h}$ at $37^{\circ} \mathrm{C}$ revealed that these cells were not fluorescent. Addition of hydrogen peroxide to the medium at a final concentration of 0.1 $\mathrm{mM}$ induced fluorescence in all myocytes that was prevented by either catalase $(33 \mathrm{U} / \mathrm{ml})$ or desferroxamine $(0.1 \mathrm{mM})$, and addition of the superoxide generating system xanthine and xanthine oxidase also induced fluorescence that was prevented by addition of superoxide dismutase $(200 \mathrm{U} / \mathrm{ml})$ and catalase ( 33 $\mathrm{U} / \mathrm{ml}$ ). Adherent neutrophils also induced myocyte fluorescence. Within $5 \mathrm{~min}$ after the adherence of ZAS-stimulated neutrophils, fluorescence developed in individual myocytes with attached neutrophils, and within an additional 1-5 min, sustained contracture of the fluorescent myocytes was often seen. In $>100$ observations of neutrophil adherence-dependent oxidation, fluorescence always preceded or coincided with contracture. Initially, the highest intensity fluorescence was observed at the site of an attached neutrophil (Fig. 6) progressing to high intensity fluorescence throughout the cell usually within $<5$ min. Myocytes without adherent neutrophils were not fluorescent and rarely contracted during the observation period. These experiments were repeated with IL-6 and rTNF $\alpha$ stimulated myocytes with equivalent results (data not shown).

When only neutrophils were loaded with DCFH, fluorescence following adhesion to the myocytes was seen only in the neutrophils (Fig. 7). In addition, this fluorescence was restricted to adherent neutrophils. The level of chemotactic stimulus needed to promote neutrophil adherence to cytokine-stimulated myocytes failed to induce fluorescence in nonadherent neutrophils. This adherence-dependent fluorescence in loaded

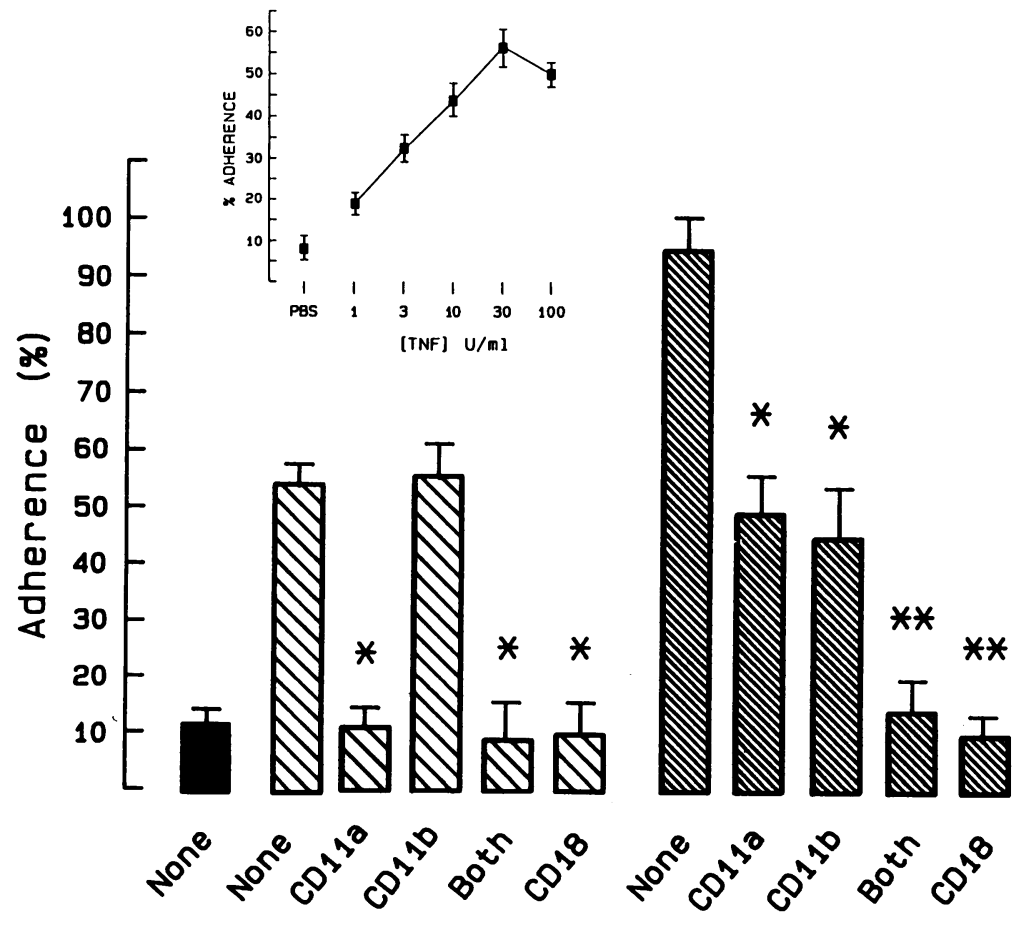

Figure 3. Stimulation of CJVEC adhesiveness for canine neutrophils by rTNF $\alpha$. The inset graph demonstrates the effects of incubating CJVEC monolayers for $4 \mathrm{~h}$ in different concentrations of rTNF $\alpha$ on the adhesion of isolated and previously unstimulated canine neutrophils. Adhesion was determined under static conditions after allowing neutrophils to contact the monolayers for 500 s. Based on this dose-response study, $30 \mathrm{U} / \mathrm{ml}$ of rTNF $\alpha$ was selected for subsequent experiments. Adhesion of previously unstimulated canine neutrophils to TNF-stimulated monolayers (coarse cross-hatched bars) is shown. In addition, adhesion of PAF-stimulated ( 100 $\mathrm{ng} / \mathrm{ml}, 5 \mathrm{~min}$ ) canine neutrophils to TNF-stimulated monolayers (fine cross-hatched bars) is also shown. None indicates that antibody was not added. The antigen recognized by the mAbs appears under the bars, and the specific $\mathrm{mAbs}$ used were: CD1 1a, R7.1 (10 $\mu \mathrm{g} / \mathrm{ml}, \mathrm{IgG})$; CD1 1b, MY904 ( $10 \mu \mathrm{g} / \mathrm{ml}$, IgG); CD18, R15.7 (10 $\mu \mathrm{g} /$ $\mathrm{ml}, \mathrm{IgG})$. Both indicates that R7.1 and MY904 were both added to the neutrophil suspension. ${ }^{*} P<0.01$ compared to the appropriate control condition without added $\mathrm{mAb}$; ${ }^{* *} P<0.01$ compared to either anti-CD1 la or antiCD11b; $n=5$. 


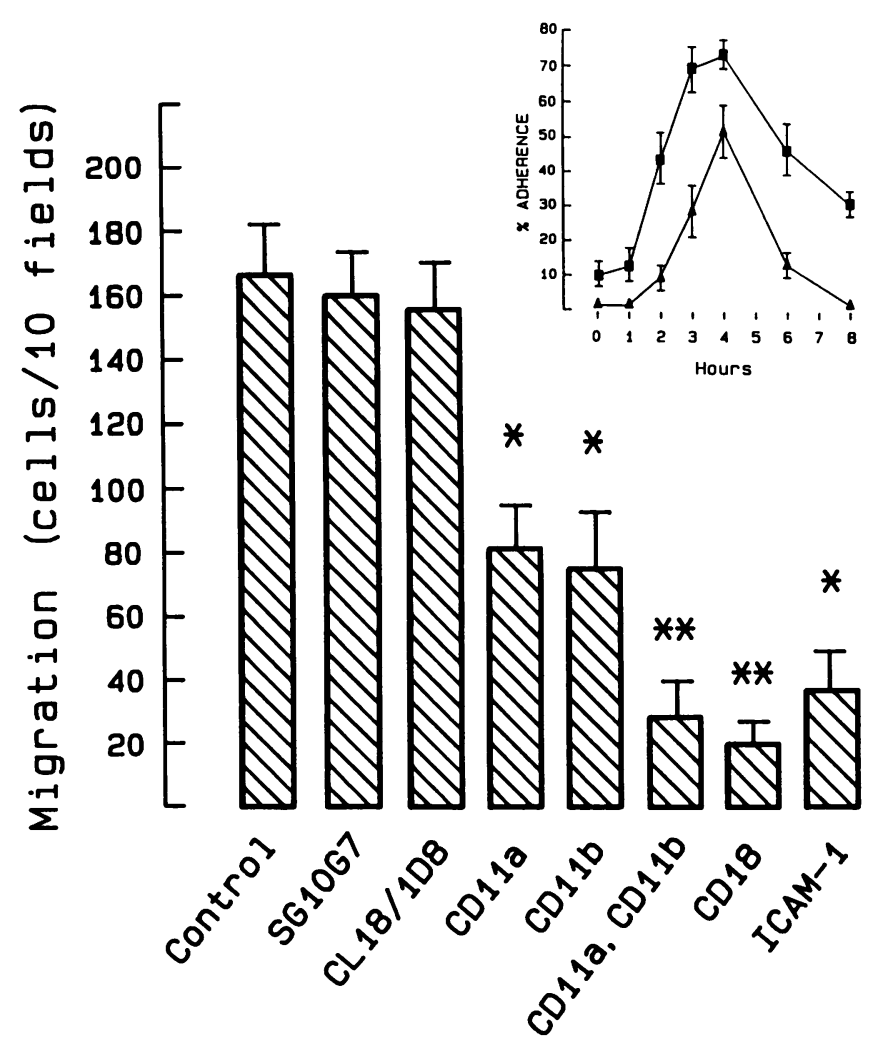

Figure 4. Transendothelial migration of canine neutrophils. The inset graph demonstrates the time course for adhesion (upper curve) of previously unstimulated neutrophils to CJVEC monolayers stimulated with LPS $(10 \mathrm{ng} / \mathrm{ml})$, and the proportion of the adherent cells that migrated beneath the endothelial monolayer (lower curve). Using conditions found to result in maximum transendothelial migration $(4 \mathrm{~h})$, the effects of various mAbs on migration were assessed. When mAbs were present, they were incubated with the appropriate cells $15 \mathrm{~min}$ before the migration assay and retained with the cells throughout the period of time allowed for migration $(1,000 \mathrm{~s})$. The following $\mathrm{mAbs}$ were used in these experiments and recognize the antigens listed below the bars: CD1 1a, R7.1 (10 $\mu \mathrm{g} / \mathrm{ml}, \mathrm{IgG})$; CD1 1b, MY904 ( $10 \mu \mathrm{g} / \mathrm{ml}, \mathrm{IgG}) ; \mathrm{CD} 18, \mathrm{R} 15.7$ ( $10 \mu \mathrm{g} / \mathrm{ml}, \mathrm{IgG})$; ICAM-1, $\mathrm{CL} 18 / 6\left(20 \mu \mathrm{g} / \mathrm{ml}, \mathrm{F}(\mathrm{ab})_{2}^{\prime}\right) .{ }^{*} P<0.01, n=4$, compared with control condition; ${ }^{* *} P<0.01, n=5$, compared to conditions with either anti-CD1 la or anti-CD11b. Two binding control MAbs were also used: SG10G7, anti-canine neutrophil, antigen unknown; CL18/ 1D8, anti-canine ICAM-1, recognizes a nonfunctional epitope.

neutrophils appeared more rapidly after adhesion (within seconds) than that seen with DCFH-loaded myocytes.

Specific adhesion molecules and intracellular oxidation. Only conditions that promoted neutrophil-myocyte adhesion resulted in fluorescence of DCFH-loaded myocytes (i.e., when both neutrophils and myocytes were stimulated with chemotactic factors and cytokines, respectively) (Fig. 8), and the anti-CD18 mAb, R15.7, blocked adhesion and myocyte fluorescence. Adhesion and fluorescence were also blocked by antiICAM-1 and anti-CD11b mAbs, but anti-CD11a mAb R7.1 blocked neither adherence nor fluorescence (Fig. 9). These results indicate that both adherence and fluorescence depend primarily on Mac-1 and ICAM-1.

Characteristics of neutrophil-induced myocyte oxidation. Increasing fluorescence indicating intracellular oxidation of cardiac myocytes resulting from neutrophil adherence occurred much more rapidly than hydrogen peroxide was de- tected in the extracellular medium in our previous studies of neutrophil-myocyte CD18-dependent adhesion (7). Fluorescence of DCFH loaded myocytes was seen within 5 min after neutrophil adherence under fluorescence microscopy (Fig. 5), and could be detected within $15 \mathrm{~min}$ after addition of ZAS in the fluorometric assay (Figs. 8 and 9).

Various antioxidants were used to further characterize the chemical nature of the myocyte DCFH oxidizing event, and to determine if myocyte fluorescence was the result of reactive oxygen species initially compartmentalized within myocytes. Desferroxamine and dimethylthiourea, antioxidants known to enter cells $(27,28)$, eliminated fluorescence without affecting adhesion (Table II and Fig. 10), and completely prevented adherence-dependent contracture (data not shown). In contrast, when SOD, catalase or starch-immobilized desferroxamine, antioxidants that do not enter cells, were added to the medium containing cytokine-stimulated, DCFH-loaded myocytes before chemotactic stimulation of the neutrophils, they failed to inhibit either the adherence of neutrophils to myocytes caused by chemotactic stimulation, or the associated increases in fluorescence (Fig. 10 and Table II). These data indicated that the adherence-dependent transfer of reactive oxygen species was tightly compartmented and not in equilibrium with the extracellular medium during the 30-min observation period. Efforts to detect extracellular superoxide under the adhesive conditions that yielded maximum fluorescence of DCFHloaded myocytes were negative over the same time period (Fig. 11 ) in agreement with our earlier studies (7). In contrast, addition of PMA $(250 \mathrm{ng} / \mathrm{ml})$, resulted in the rapid appearance of extracellular superoxide (Fig. 11).

\section{Discussion}

Previous studies of acute myocardial infarction in animal models indicate that the stimuli needed to promote the adhesive and oxidative events observed in vitro in this report are present within the first few hours after reperfusion of the ischemic myocardium. Several reports provide compelling evidence that complement activation follows myocardial ischemia (29-35), and plays a significant role in tissue injury (36). Postischemic cardiac lymph is chemotactic for neutrophils for up to $4 \mathrm{~h}$ after the initiation of reperfusion (11), and the rate of neutrophil accumulation in the ischemic myocardium is greatest during this period (1). Recent evidence indicates that a predominant chemotactic factor present in this lymph is C5a (37), a finding that is not surprising in light of two earlier studies showing that $\mathrm{Clq}$ binding proteins capable of activating the classical complement pathway egress from the injured myocardium, and that $\mathrm{Clq}$ localization is seen at the same sites where neutrophil localization occurs during the first few hours of reperfusion $(30,31)$. In recent studies, we have found that postischemic cardiac lymph collected within the first hour after the initiation of reperfusion stimulates isolated cardiac myocytes to express ICAM-1 and thereby become more adhesive to chemotactically stimulated neutrophils $(8,9)$. The cytokine predominantly involved in this effect of postischemic cardiac lymph is IL-6 (9), and evidence of cytokine activity persists for up to 72 $h$. Thus, within the first few hours of reperfusion, factors (predominantly C5a and IL-6) are present within the extracellular space that could lead to neutrophil-myocyte adhesion and neutrophil-induced myocyte intracellular oxidative stress. 


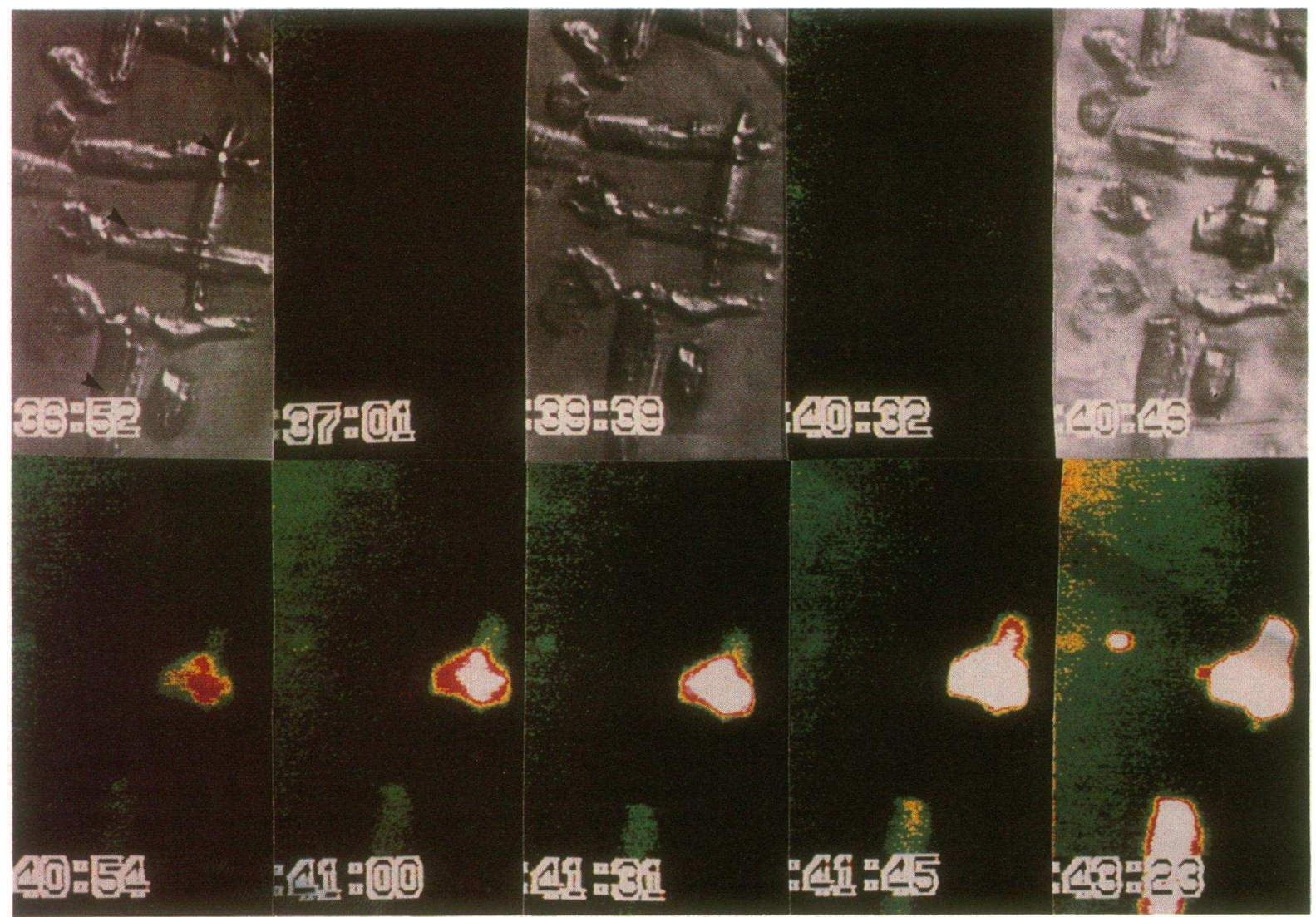

Figure 5. Time lapse photographs of fluorescence in myocytes. Adult canine myocytes were isolated, preincubated with $4 \mathrm{U} / \mathrm{ml} \mathrm{rIL}-1 \beta$ for $3 \mathrm{~h}$, loaded with DCFH for $20 \mathrm{~min}$ at $37^{\circ} \mathrm{C}$, and washed twice. Loaded myocytes were suspended with neutrophils at a ratio of $\sim 1: 50$ in complete medium containing $1 \%$ ZAS as a chemotactic stimulus. Coverslip preparations were made and changes in cell shape and fluorescence were recorded using a computer aided image analysis system. The time shown is minutes:seconds from an arbitrary time on video tape. Bound neutrophils are indicated with arrows on the first frame. Note that fluorescence in the upper myocytes begins at 40:32, and by 40:46 contracture is noted. Fluorescence begins in the lower myocytes at $40: 54$ and contracture begins by $43: 23$, while severe contracture occurred by $45: 30$ (not shown).

The results of the present studies indicated that the transfer of reactive oxygen species from the neutrophil to the myocyte appears to be localized to the intracellular compartment, and

requires $\beta 2$ integrin and ICAM-1-dependent adherence. The precise mechanism and oxidative products involved in the oxidation of the DCFH probe are not specifically elucidated in the
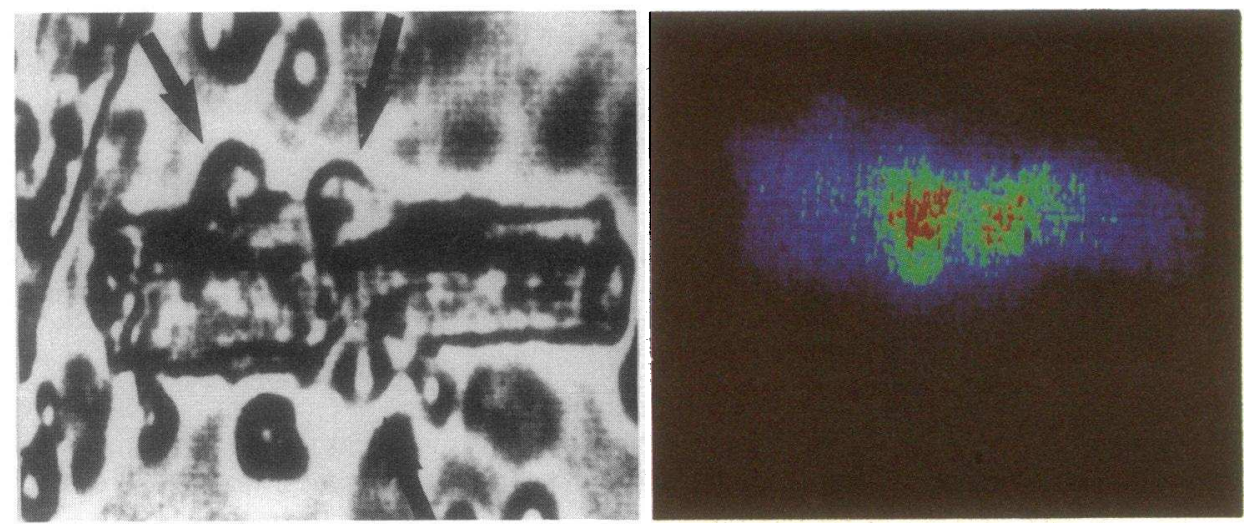

Figure 6. Cardiac myocyte fluorescence near the site of neutrophil adherence. Adult canine myocytes were isolated, preincubated with 4 $\mathrm{U} / \mathrm{ml} \mathrm{rIL}-1 \beta$ for $3 \mathrm{~h}$, loaded with DCFH for $20 \mathrm{~min}$ at $37^{\circ} \mathrm{C}$ and washed twice. Loaded myocytes were suspended with neutrophils at a ratio of $\sim 1: 50$ in complete medium containing $1 \%$ ZAS as a chemotactic stimulus. Coverslip preparations were made, and changes in fluorescence were recorded using a computer-aided image analysis system. A myocyte to which two neutrophils were bound was examined during the first minute of neutrophil adherence. The phase contrast image is shown on the left and the corresponding fluorescence image is shown at the right. 

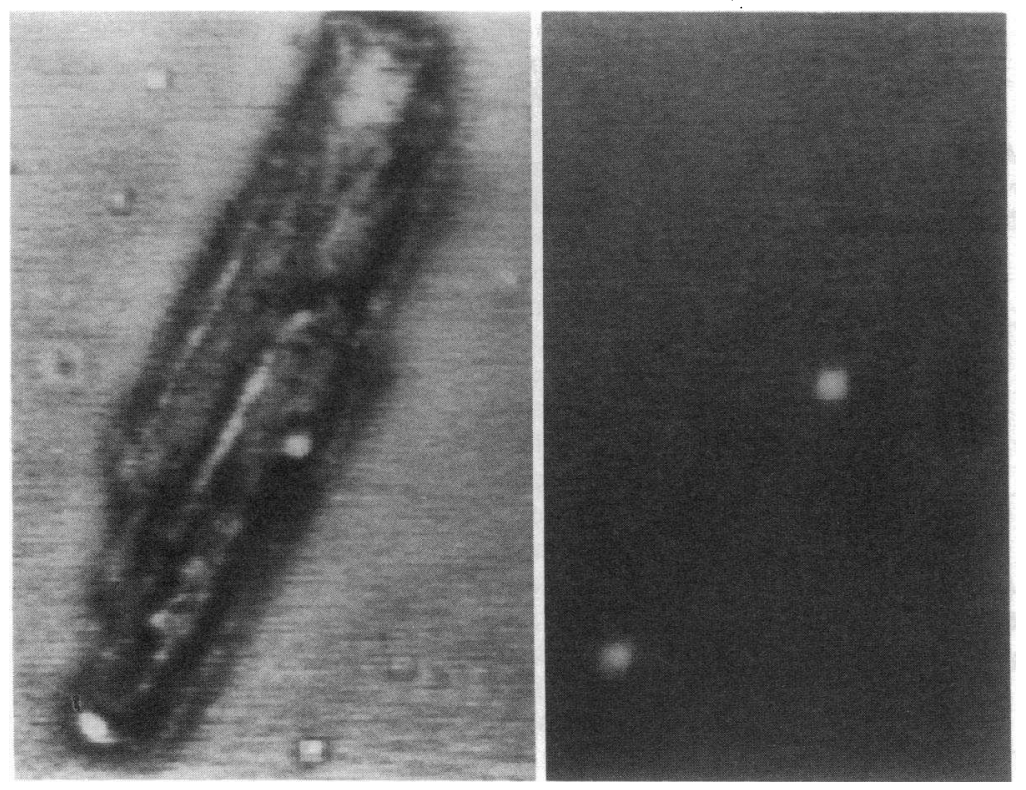

Figure 7. Fluorescence of DCFH-loaded neutrophils following adherence to myocytes. Neutrophils were loaded with DCFH and washed twice. Myocytes were suspended with neutrophils at a ratio of $\sim 1: 50$ in complete medium containing $1 \%$ ZAS as a chemotactic stimulus. Coverslip preparations were made and changes in cell shape and fluorescence were recorded using a computeraided image analysis system. Although neutrophil:myocyte ratios were $\sim 50: 1$, the only fluorescence seen coincides with neutrophils adhering to the surface of the myocytes as shown in this example.

present studies. However, iron appears to be necessary, and fluorescence can be induced by the addition of extracellular superoxide or hydrogen peroxide. These findings suggest the possible involvement of a Haber-Weiss reaction generating hydroxyl radical intermediates. This hypothesis is further supported by the marked effectiveness of dimethylthiourea, a hydroxyl radical scavenger (38), in preventing fluorescence and myocardial contracture. Hence, generation of lipid hydroperoxides in an iron-dependent manner may contribute to DCFH fluorescence since this probe can be oxidized by a variety of reactive oxygen species. It appears that myocyte injury induced by adherent neutrophils relates to oxidative injury since interventions blocking fluorescence prevented contracture during the 1-h observation period.
Several investigations under related conditions have revealed that the adherence-dependent oxidative burst of neutrophils involving $\beta 2$ integrins $(15,39)$ is characterized by a prolonged lag phase lasting $\sim 1 \mathrm{~h}(40,41)$. These studies have evaluated extracellular release of hydrogen peroxide after stimulation of either canine or human neutrophils adherent to endothelial cells or protein-coated plastic. In our earlier studies with canine neutrophils adherent to isolated canine cardiac myocytes, we found a similar lag phase when using an extracellular detection system to quantitate hydrogen peroxide. However, the results in the present study indicate that the respiratory burst begins rapidly after CD18-dependent adhesion to cytokine-stimulated myocytes occurs, but the reactive oxygen is localized to the intracellular compartment. The target is
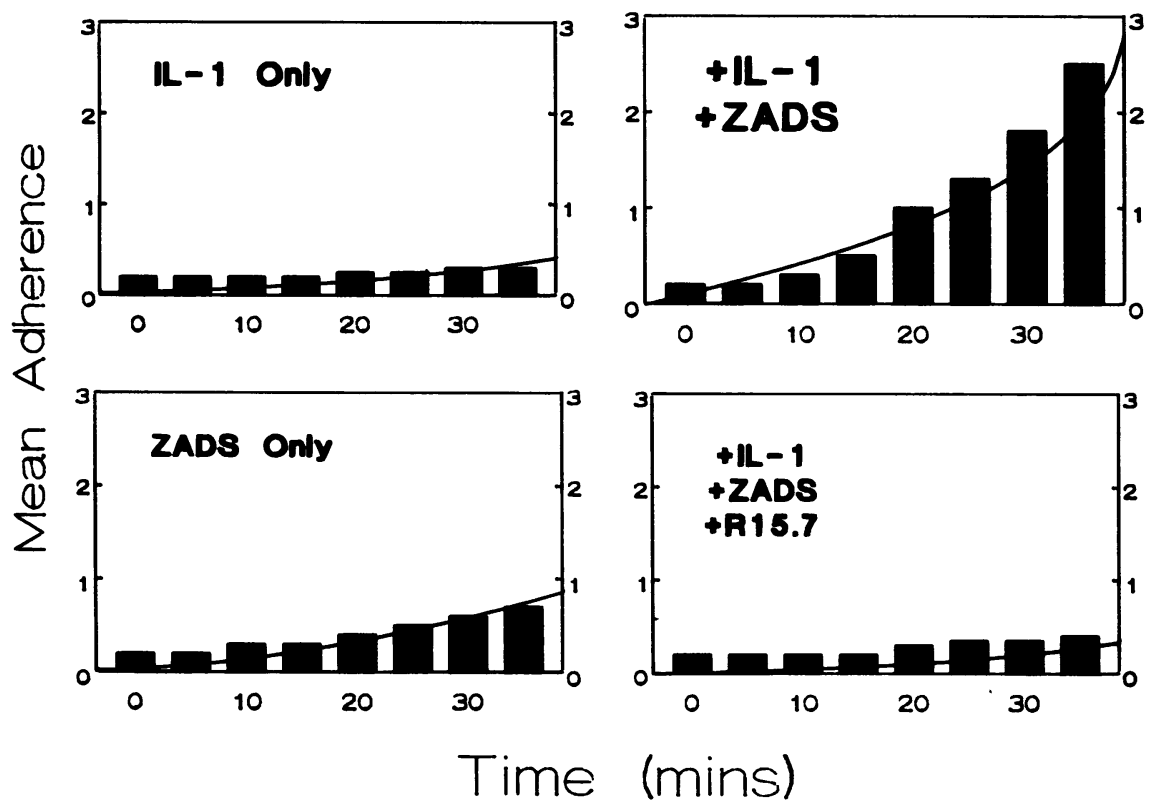

Figure 8. Relationship of neutrophil adherence to myocyte fluorescence. Adult canine myocytes were isolated, preincubated with $4 \mathrm{U} / \mathrm{ml}$ rIL- $1 \beta$ for $3 \mathrm{~h}$, loaded with DCFH for $20 \mathrm{~min}$ at $37^{\circ} \mathrm{C}$, and washed twice. Loaded myocytes were suspended with neutrophils at a ratio of $\sim 1: 50$ in complete medium. An aliquot of the cell suspension was placed in a cuvette, and ZAS was added to stimulate neutrophils at time 0 where indicated. Samples of the cell suspension were evaluated for neutrophil-myocyte adherence (expressed as mean number of neutrophils per myocyte, bars), and fluorescence was determined using a fluorometer (PerkinElmer Cetus Instruments; excitation wavelength, $488 \mathrm{~nm}$, and emission wavelength, $521 \mathrm{~nm}$; result plotted as line). $R 15.7(50 \mu \mathrm{g} / \mathrm{ml})$ was present throughout the incubation. Results are from a single experiment are presented and are representative of five experiments with different myocyte and neutrophil preparations. 

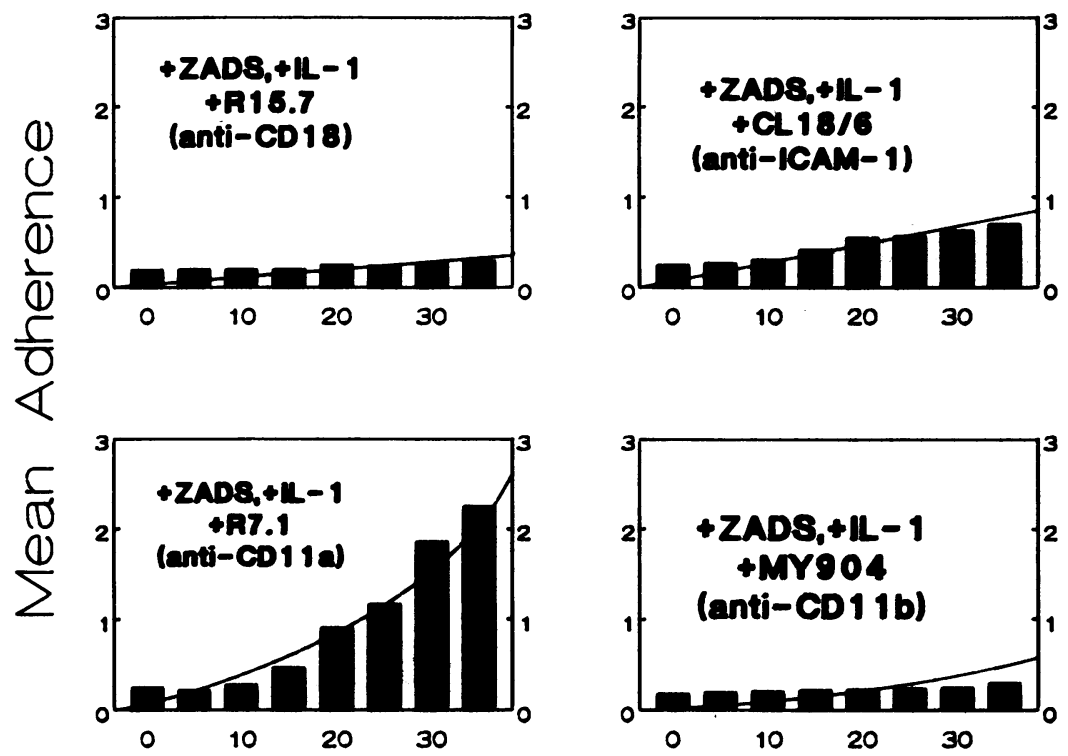

Time (mins)
Figure 9. Neutrophil/myocyte adherence and myocyte fluorescence: effects of monoclonal antibodies against CD11a, CD11b, ICAM-1. Adult canine myocytes were isolated, preincubated with $4 \mathrm{U} / \mathrm{ml} \mathrm{rIL-} 1 \beta$ for $3 \mathrm{~h}$, loaded with DCFH for $20 \mathrm{~min}$ at $37^{\circ} \mathrm{C}$, and washed twice. Loaded myocytes were suspended with neutrophils at a ratio of $\sim 1: 50$ in complete medium. An aliquot of the cell suspension was placed in a cuvette, and ZAS was added to stimulate neutrophils at time 0 where indicated. Samples of the cell suspension were evaluated for neutrophil-myocyte adherence (expressed as mean number of neutrophils per myocyte, bars), and fluorescence was determined using a fluorometer (Perkin-Elmer Cetus Instruments; excitation wavelength, $\mathbf{4 8 8}$ $\mathrm{nm}$, and emission wavelength, $521 \mathrm{~nm}$; result plotted as line). R7.1 (anti-CD11a, $100 \mu \mathrm{g} /$ $\mathrm{ml}), \mathrm{MY} 904 \mathrm{~F}(\mathrm{ab})_{2}^{\prime}(20 \mu \mathrm{g} / \mathrm{ml})$ or CL18/6 $\mathrm{F}(\mathrm{ab})_{2}^{\prime}(20 \mu \mathrm{g} / \mathrm{ml})$ were present throughout the incubation period. Results are from a single experiment and representative of five experiments with different myocyte and neutrophil preparations. thereby exposed to toxic neutrophil-derived products at a high concentration at the neutrophil-myocyte interface in a manner inaccessible to extracellular antioxidants. Similar phenomena have been described in the past with regard to compartmental-

Table II. Effects of Monoclonal Antibodies and Antioxidants on Canine Neutrophil Adherence to Isolated Canine Myocytes and DCFH Fluorescence

\begin{tabular}{|c|c|c|c|}
\hline \multirow[b]{2}{*}{ Experimental conditions* } & & \multicolumn{2}{|c|}{ Percent inhibition } \\
\hline & & Fluorescence $e^{\ddagger}$ & Adherences \\
\hline & $n$ & & \\
\hline Anti-CD18 (R15.7) & 5 & $95.6 \pm 2.7^{\| \prime}$ & $92.5 \pm 2.2^{11}$ \\
\hline Anti-ICAM-1 (CL18/6) & 5 & $71.7 \pm 9.7^{\| 1}$ & $84.3 \pm 3.4^{\prime \prime}$ \\
\hline Anti-CD1 1b (MY904) & 5 & $82.0 \pm 2.1^{11}$ & $86.3 \pm 3.4^{\prime \prime}$ \\
\hline Anti-CD11a (R7.1) & 5 & None & $0.5 \pm 0.5$ \\
\hline SOD + catalase & 5 & $5.0 \pm 5.9$ & None \\
\hline Desferroxamine & 4 & $100^{\text {lin }}$ & $1.0 \pm 1.0$ \\
\hline $\begin{array}{l}\text { Starch-immobilized } \\
\text { desferroxamine }\end{array}$ & 3 & None & None \\
\hline Dimethylthiourèa & 4 & $100^{\mathrm{min}}$ & $0.6 \pm 0.3$ \\
\hline
\end{tabular}

* Isolated canine cardiac myocytes were stimulated for $3 \mathrm{~h}$ with rIL$1 \beta$ and then loaded with DCFH for $20 \mathrm{~min}$, washed, and incubated with neutrophils in the presence of $1 \%$ zymosan activated serum for $30 \mathrm{~min}$ at $37^{\circ} \mathrm{C}$. The reagents listed below were present in the cell suspension throughout the incubation period. $\mathrm{mAbs}$ were used at 20 $\mu \mathrm{g} / \mathrm{ml}$; SOD, $200 \mathrm{U} / \mathrm{ml}$; catalase, $33 \mathrm{U} / \mathrm{ml}$; desferroxamine, $0.1 \mathrm{mM}$; and dimethylthiourea, $0.1 \mathrm{mM}$. ${ }^{\ddagger}$ Fluorescence was determined at the end of the 30-min incubation period using a fluorometer. ${ }^{8} \mathrm{Ad}-$ herence was determined visually under phase contrast microscopy by counting the number of neutrophils attached to 100 myocytes. " $P<0.01$. 'In each experiment, fluorescence was completely inhibited. ization of protein at adhesive interfaces $(42,43)$. Thus under conditions of cell-cell adhesion the adherence-dependent respiratory burst appears to have two phases, one that localizes metabolic events at the adhesive interface, and a subsequent phase where reactive oxygen is detected in the extracellular space. This latter phase has been studied most extensively with neutrophils adherent to protein-coated plastic (and therefore Mac-1 dependent) where it may depend in part on a delay in sustained adhesion and cell spreading (44), and appears to be linked to the modulations of intracellular regulatory mechanisms seen by Nathan et al. (44) and Shappell et al. (45).

The results in this report also show for the first time that ICAM- 1 can serve as a ligand for the adherence-dependent neutrophil respiratory burst. Previous studies have linked ICAM-1 to neutrophil adhesion and transendothelial migration $(8,10,12,13,18,22,25)$ at the vessel wall, but our results indicate that ICAM-1 may be linked to cytotoxic behavior accompanying neutrophil/parenchymal cell adhesion. The distinctions between neutrophil/endothelial interactions and neutrophil/cardiac myocyte interactions are particularly interesting. Human and canine neutrophils have been shown to use both LFA-1 and Mac-1 in their interactions with cytokine-stimulated endothelial cells, and this interaction is characterized by a high degree of neutrophil mobility $(10,25,46)$ where neutrophils migrate rapidly through endothelial monolayers (18, 22, $25,47-49)$. In contrast, canine neutrophil adherence to cytokine-stimulated cardiac myocytes appears to be more prolonged and is apparently heavily dependent on Mac-1. The use of mAb R7.1 (anti-CD1 la) indicated that LFA-1 is not a significant determinant of this neutrophil/myocyte adhesion. This conclusion is supported by the finding that this $\mathrm{mAb}$ was capable of blocking canine neutrophil/endothelial interactions that are analogous to those previously shown to be LFA-1 dependent with human cells $(10,22,25,26)$.

ICAM-1 densely inserted in planar artificial membranes has been shown to support a high degree of binding of isolated 


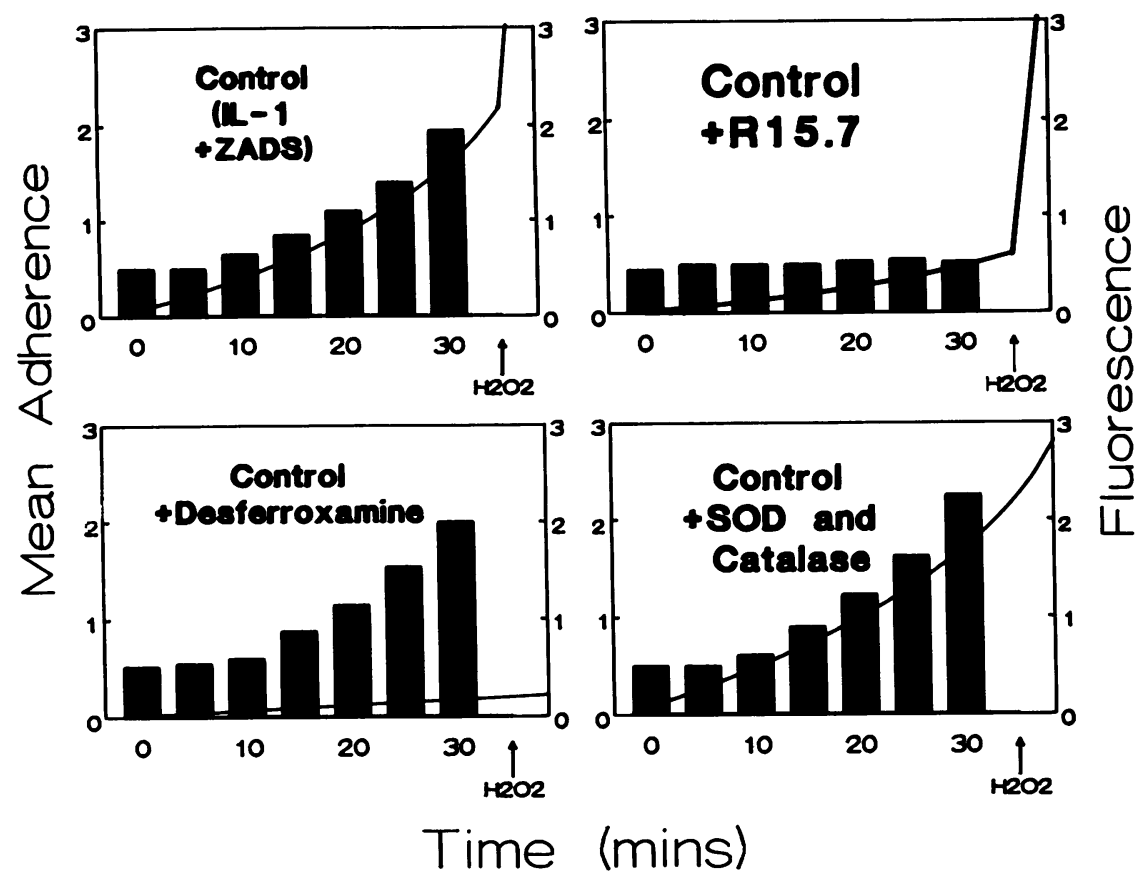

Figure 10. Effect of antioxidant strategies on adherence and fluorescence. Adult canine myocytes were isolated, preincubated with 4 $\mathrm{U} / \mathrm{ml} \mathrm{rIL-} 1 \beta$ for $3 \mathrm{~h}$, loaded with DCFH for $20 \mathrm{~min}$ at $37^{\circ} \mathrm{C}$, and washed twice. Loaded myocytes were suspended with neutrophils at a ratio of $\sim 1: 50$ in complete medium. An aliquot of the cell suspension was placed in a cuvette, and ZAS was added to stimulate neutrophils at time 0 where indicated. Samples of the cell suspension were evaluated for neutrophil-myocyte adherence (expressed as mean number of neutrophils per myocyte, bars), and fluorescence was determined using a fluorometer (Perkin-Elmer Cetus Instruments; excitation wavelength, $488 \mathrm{~nm}$, and emission wavelength, $521 \mathrm{~nm}$; result plotted as line). SOD $(200 \mathrm{U} / \mathrm{ml})$, catalase $(33 \mathrm{U} / \mathrm{ml})$, desferroxamine $(0.1 \mathrm{mM})$, or anti-CD18 mAb (R15.7, $50 \mu \mathrm{g} / \mathrm{ml})$ were present from time 0 where indicated. Hydrogen peroxide $(0.1 \mathrm{mM})$ was added to all four conditions at $35 \mathrm{~min}$ (arrow). Note that while addition of SOD and catalase at concentrations sufficient to prevent fluorescence induced by adding of hydrogen peroxide to DCFH-loaded myocytes without added

neutrophils (data not shown) did not alter adherence or adherence-dependent fluorescence, it did prevent hydrogen peroxide induced fluorescence. Desferroxamine did not alter adherence but prevented fluorescence resulting from either neutrophil adherence or exogenous hydrogen peroxide. Results are from a single experiment and are representative of five experiments with different myocyte and neutrophil preparations.

neutrophils exposed to chemotactic factors, and anti-CD11b and anti-CD1 la mAbs each reduce this adherence by $\sim 50 \%$ $(10,50)$. Diamond et al. (24) more directly assessed the binding of CD11b/CD18 to ICAM-1 by showing that COS cells transfected with both cDNAs for CD18 and CD1 $1 \mathrm{~b}$ adhered to

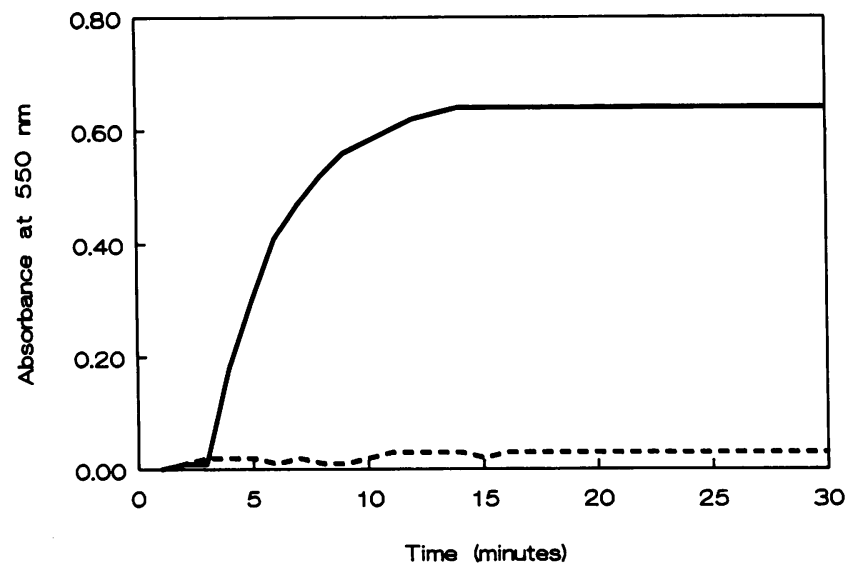

Figure 11. Superoxide release in the external media during neutrophil/myocyte adherence. Adult canine myocytes were isolated and preincubated with $4 \mathrm{U} / \mathrm{ml}$ rIL-1 $\beta$ for $3 \mathrm{~h}$. Stimulated myocytes were suspended with neutrophils at a ratio of $\sim 1: 50$ in complete medium. An aliquot of the cell suspension was placed in a cuvette, and ZAS was added to stimulate neutrophil adherence. Cytochrome $c(10 \mu \mathrm{M})$ was used to detect extracellular superoxide (absorbance was monitored at wavelength of $550 \mathrm{~nm}$ ). These cell preparations did not release superoxide (dashed line). In contrast, when such cell preparations were incubated with phorbol myristate acetate $(250 \mathrm{ng} / \mathrm{ml})$ in place of ZAS, superoxide was readily detected in the extracellular medium (solid line). isolated ICAM-1, and that anti-ICAM-1 monoclonal antibody, R6.5 (18), was almost completely inhibitory. In the reciprocal experiment $\mathrm{COS}$ cells and human umbilical vein endothelial cells expressing ICAM-1 bound to planar membranes containing isolated $\mathrm{CD} 1 \mathrm{~b} / \mathrm{CD} 18$ heterodimer. The interaction of CD11b/CD18 and CD11a/CD18 with ICAM-1 was not found to be identical, though. mAbs against different domains of ICAM-1 exhibited differential inhibition of these interactions (e.g., mAb R6.5 (18) recognizes domain 2 and inhibits interactions with both Mac-1 and LFA-1, while mAb LB2 (50) recognizes domain 1 and inhibits only interactions with LFA-1. Furthermore, using domain-deleted and chimeric forms of ICAM1, Staunton et al. (51) demonstrated that CD1 1a/CD18 bound to the first domain of ICAM-1, and Diamond et al. (24) demonstrated that $\mathrm{CD} 11 \mathrm{~b} / \mathrm{CD} 18$ bound to the third domain of ICAM-1. Diamond et al. (24) found that the size of the Nlinked oligosaccharide chain on domain 3 of ICAM-1 influenced the interaction between CD11b/CD18 and ICAM-1. Adhesion depended primarily on Mac-1 if ICAM-1 had a smaller N-linked side chain, but on LFA-1 if ICAM-1 had a complex carbohydrate side chain. ICAM-1 from different cell types may differ in the level and type of $\mathrm{N}$-linked carbohydrates (52), and thereby may regulate the interactions of different cells with neutrophils. It remains to be determined why the interaction of chemotactically stimulated neutrophils with ICAM-1 on cardiac myocytes is predominantly Mac-1 dependent, but the work of Diamond et al. (24) raises the possibility that glycosylation of cardiomyocyte ICAM-1 may favor Mac1-dependent adhesion more than that of endothelial ICAM-1. The potential pathophysiologic significance of a predominantly Mac-1-dependent adhesion is underscored by the ability of this $\beta 2$ integrin to promote a pronounced respiratory burst, an outcome apparently not supported by LFA-1 (15). 


\section{Acknowledgments}

The authors acknowledge the excellent technical assistance of B. J. Hughes, Shirley Williams, and Barbara Leone, the excellent secretarial assistance of Michelle Swarthout, and helpful discussions with Dr. Roger Rossen and Dr. W. J. Dreyer.

This study was supported in part by grants HL-42550, HL-41408, AI-28071, AI-19031, and HL-47163 from the National Institutes of Health, and a grant from Boehringer Ingelheim Pharmaceuticals, Inc.

\section{References}

1. Dreyer, W. J., L. H. Michael, M. S. West, C. W. Smith, R. Rothlein, R. D. Rossen, D. C. Anderson, and M. L. Entman. 1991. Neutrophil accumulation in ischemic canine myocardium: insights into the time course, distribution, and mechanism of localization during early reperfusion. Circulation. 84:400-411.

2. Mullane, K. M., and C. W. Smith. 1990. The role of leukocytes in ischemic damage, reperfusion injury and repair of the myocardium. In Pathophysiology of Severe Ischemic Myocardial Injury. H. M. Piper, editor. Kluwer Academic Publishers, Dordrecht. 239-267.

3. Smith, E. F., J. W. Egan, P. J. Bugelshi, L. M. Hillegass, and D. E. Hill. 1988. Temporal relation between neutrophil accumulation and myocardial reperfusion. Am. J. Physiol. 255:H1060-H1068.

4. Engler, R. L., G. W. Schmid-Schonbein, and R. S. Parelec. 1983. Leukocyte capillary plugging in myocardial ischemia and reperfusion in the dog. Am. J. Pathol. 111:98-111.

5. Schmid-Schonbein, G. W. 1987. Capillary plugging by granulocytes and the no-reflow phenomenon in the microcirculation. Fed. Proc. 46:2397-2401.

6. Schmid-Schonbein, G. W., and R. L. Engler. 1987. Granulocytes as active participants in acute myocardial ischemia and infarction. Am. J. Cardiovasc. Pathol. 1:15-30.

7. Entman, M. L., K. Youker, S. B. Shappell, C. Siegel, R. Rothlein, W. J. Dreyer, F. C. Schmalstieg, and C. W. Smith. 1990. Neutrophil adherence to isolated adult canine myocytes: evidence for a CD18-dependent mechanism. $J$. Clin. Invest. 85:1497-1506.

8. Smith, C. W., M. L. Entman, C. L. Lane, A. L. Beaudet, T. I. Ty, K. Youker, H. K. Hawkins, and D. C. Anderson. 1991. Adherence of neutrophils to canine cardiac myocytes in vitro is dependent on intercellular adhesion molecule1. J. Clin. Invest. 88:1216-1223.

9. Youker, K., C. W. Smith, D. C. Anderson, D. Miller, L. H. Michael, R. D. Rossen, and M. L. Entman. 1991. Neutrophil adherence to isolated adult cardiac myocytes: induction by cardiac lymph collected during ischemia and reperfusion. J. Clin. Invest. 89:602-609.

10. Smith, C. W., S. D. Marlin, R. Rothlein, C. Toman, and D. C. Anderson. 1989. Cooperative interactions of LFA-1 and Mac-1 with intercellular adhesion molecule- 1 in facilitating adherence and transendothelial migration of human neutrophils in vitro. J. Clin. Invest. 83:2008-2017.

11. Dreyer, W. J., C. W. Smith, L. H. Michael, R. D. Rossen, B. J. Hughes, M. L. Entman, and D. C. Anderson. 1989. Canine neutrophil activation by cardiac lymph obtained during reperfusion of ischemic myocardium. Circ. Res. 65:1751-1762.

12. Argenbright, L. W., L. G. Letts, and R. Rothlein. 1991. Monoclonal antibodies to the leukocyte membrane CD18 glycoprotein complex and to intercellular adhesion molecule-1 inhibit leukocyte-endothelial adhesion in rabbits. $J$. Leukocyte Biol. 49:253-257.

13. Argenbright, L. W., and R. W. Barton. 1992. Interactions of leukocyte integrins with intercellular adhesion molecule 1 in the production of inflammatory vascular injury in vivo. The Shwartzman reaction revisited. J. Clin. Invest. 89:259-272.

14. Jaeschke, H., A. I. Farhood, and C. W. Smith. 1991. Neutrophil-induced liver cell injury in endotoxin shock is a CD11b/CD18-dependent mechanism. Am. J. Physiol. 261:c-1051-c-1056.

15. Shappell, S. B., C. Toman, D. C. Anderson, A. A. Taylor, M. L. Entman, and C. W. Smith. 1990. Mac-1 (CD1 1b/CD18) mediates adherence-dependent hydrogen peroxide production by human and canine neutrophils. J. Immunol. 144:2702-2711.

16. Anderson, D. C., K. L. B. Freeman, B. Heerdt, B. J. Hughes, R. M. Jack, and C. W. Smith. 1987. Abnormal stimulated adherence of neonatal granulocytes: impaired induction of surface Mac-1 by chemotactic factors or secretagogues. Blood. 70:740-750.

17. Ford, J. W., W. E. Burkel, and R. H. Kahn. 1981. Isolation of adult canine venous endothelium for tissue culture. In Vitro (Rockville). 17:44-50.

18. Smith, C. W., R. Rothlein, B. J. Hughes, M. M. Mariscalco, F. C. Schmalstieg, and D. C. Anderson. 1988. Recognition of an endothelial determinant for CD18-dependent human neutrophil adherence and transendothelial migration. J. Clin. Invest. 82:1746-1756.

19. Shappell, S. B., A. A. Taylor, H. Hughes, J. R. Mitchell, D. C. Anderson, and C. W. Smith. 1990. Comparison of antioxidant and nonantioxidant lipoxygenase inhibitors on neutrophil function. Implications for pathogenesis of myocardial reperfusion injury. J. Pharmacol. Exp. Ther. 252:531-538.

20. Anderson, D. C., L. J. Miller, F. C. Schmalstieg, R. Rothlein, and T. A Springer. 1986. Contributions of the Mac-1 glycoprotein family to adherence-dependent granulocyte functions: structure-function assessments employing subunit-specific monoclonal antibodies. J. Immunol. 137:15-27.

21. Dana, N., B. Styrt, J. D. Guffin, R. F. Todd, III, M. S. Klempner, and M. A. Arnaout. 1986. Two functional domains in the phagocyte membrane glycoprotein Mol identified with monoclonal antibodies. J. Immunol. 137:32593263.

22. Luscinskas, F. W., M. I. Cybulsky, J.-M. Kiely, C. S. Peckins, V. M. Davis, and M. A. Gimrone. 1991. Cytokine-activated human endothelial monolayers support enhanced neutrophil transmigration via a mechanism involving both endothelial-leukocyte adhesion molecule- 1 and intercellular adhesion molecule1. J. Immunol. 146:1617-1625.

23. Diamond, M. S., D. E. Staunton, A. R. deFougerolles, S. A. Stacker, J. Garcia-Aguilar, M. L. Hibbs, and T. A. Springer. 1990. ICAM-1 (CD54): a counter-receptor for Mac-1 (CD11b/CD18). J. Cell Biol. 111:3129-3139.

24. Diamond, M. S., D. E. Staunton, S. D. Marlin, and T. A. Springer. 1991. Binding of the integrin Mac-1 (CD1 1b/CD18) to the third immunoglobulin-like domain of ICAM-1 (CD54) and its regulation by glycosylation. Cell. 65:961-971.

25. Furie, M. B., M. C. A. Tancinco, and C. W. Smith. 1991. Monoclonal antibodies to leukocyte integrins CD1 1a/CD18 and CD1 lb/CD18 or intercellular adhesion molecule-1 (ICAM-1) inhibit chemoattractant-stimulated neutrophil transendothelial migration in vitro. Blood. 78:2089-2097.

26. Lo, S. K., G. A. Van Seventer, S. M. Levin, and S. D. Wright. 1989. Two leukocyte receptors (CD1 1a/CD18) mediate transient adhesion to endothelium by binding to different ligands. J. Immunol. 143:3325-3329.

27. Patel, K. D., G. A. Zimmerman, S. M. Prescott, R. P. McEver, and T. M. McIntyre. 1991. Oxygen radicals induce human endothelial cells to express GMP-140 and bind neutrophils. J. Cell Biol. 112:749-759.

28. Bolli, R., W.-X. Zhu, C. J. Hartley, L. H. Michael, J. E. Repine, M. L. Hess, R. C. Kubreja and R. Roberts. 1987. Attenuation of dysfunction in the post ischemic "stunned" myocardium by dimethyl urea. Circulation. 76:458-468.

29. Hill, J. H., and P. A. Ward. 1971. The phlogistic role of C3 leukotactic fragment in myocardial infarcts of rats. J. Exp. Med. 133:885-900.

30. Rossen, R. D., L. H. Michael, A. Kagiyama, H. E. Savage, G. Hanson, J. N. Reisbery, J. N. Moake, S. H. Kim, S. Weakly, E. Giannini, and M. L. Entman. 1988. Mechanism of complement activation following coronary artery occlusion: evidence that myocardial ischemia causes release of constituents of myocardial subcellular origin which complex with the first component of complement. Circ. Res. 62:572-584.

31. Rossen, R. D., J. L. Swain, L. H. Michael, S. Weakley, E. Giannini, and M. L. Entman. 1985. Selective accumulation of the first component of complement and leukocytes in ischemic canine heart muscle: a possible initiator of an extra myocardial mechanism of ischemic injury. Circ. Res. 57:119-130.

32. Hartmann, J. R., J. A. Robinson, and R. M. Gunnar. 1977. Chemotactic activity in the coronary sinus after experimental myocardial infarction: effects of pharmacologic interventions on ischemic injury. Am. J. Cardiol. 40:550-555.

33. Pinckard, R. N., M. S. Olson, P. C. Giclas, R. Terry, J. T. Boyer, and R. A. O'Rourke. 1975. Consumption of classical complement components by heart subcellular membranes in vitro and in patients after acute myocardial infarction. J. Clin. Invest. 56:740-750.

34. Frank, M. M. 1976. Complement in Current Concepts. UpJohn Pharmaceutical Co. Scope Publications, Kalamazoo, MI. 1-48.

35. Crawford, M. H., F. L. Grover, W. P. Kolb, C. A. McMahan, R. A. O'Rourke, L. M. McManus, and R. N. Pinckard. 1988. Complement and neutrophil activation in the pathogenesis of ischemic myocardial injury. Circulation. 78:1449-1458.

36. Weisman, H. F., T. Barton, M. K. Leppo, H. C. Marsh, Jr., G. R. Carson, M. F. Concino, M. P. Boyle, K. H. Roux, M. L. Weisfeldt, and D. T. Fearon. 1990. Soluble human complement receptor type 1 : in vivo inhibitor of complement suppressing post-ischemic myocardial inflammation and necrosis. Science (Wash. DC). 249:146-151.

37. Dreyer, W. J., L. H. Michael, R. D. Rossen, T. Nguyen, D. C. Anderson, C. W. Smith, and M. L. Entman. 1991. Evidence for C5a in post-ischemic canine cardiac lymph. Clin. Res. 39:271 A. (Abstr.).

38. Fox, R. B. 1984. Prevention of granulocyte-mediated oxidant lung injury in rats by a hydroxyl radical scavenger, dimethylthiourea. J. Clin. Invest. 74:1456-1462.

39. Nathan, C. F., S. Srimal, C. Farber, E. Sanchez, L. Kabbash, A. Asch, J. Gailit, and S. D. Wright. 1989. Cytokine-induced respiratory burst of human neutrophils: dependence on extracellular matrix proteins and CD1 1 /CD18 integrins. J. Cell Biol. 109:1341-1349.

40. Nathan, C. F. 1987. Neutrophil activation on biological surfaces: massive secretion of hydrogen peroxide in response to products of macrophages and lymphocytes. J. Clin. Invest. 80:1550-1560.

41. Nathan, C. F. 1989. Respiratory burst in adherent human neutrophils: 
triggering by colony-stimulating factors CSF-GM and CSF-G. Blood. 73:301306.

42. Wright, S. D., and S. C. Silverstein. 1984. Phagocytosing macrophages exclude proteins from the zones of contact with opsonized targets. Nature (Lond.). 309:24.

43. Weitz, J. I., A. J. Huang, S. L. Landman, S. C. Nicholson, and S. C. Silverstein. 1987. Elastase-mediated fibrinogenolysis by chemoattractant-stimulated neutrophils occurs in the presence of physiologic concentrations of antiproteinases. J. Exp. Med. 166:1836-1850.

44. Nathan, C., and E. Sanchez. 1990. Tumor necrosis factor and CD11/ CD18 (beta2) integrins act synergistically to lower cAMP in human neutrophils. J. Cell Biol. 111:2171-2181.

45. Shappell, S. B., A. A. Taylor, H. Hughes, and C. W. Smith. 1991. Neutrophil CD18-mediated adherence-dependent $\mathrm{H}_{2} \mathrm{O}_{2}$ production is accompanied by diacylglycerol accumulation. J. Cell Biochem. (Suppl.) 15C:179a. (Abstr.).

46. Smith, C. W. 1992. Transendothelial migration. In Adhesion: Its Role in Inflammatory Disease. J. M. Harlan and D. Y. Liu, editors. W. H. Freeman and Co., New York. 85-115.

47. Moser, R., B. Schleiffenbaum, P. Groscurth, and J. Fehr. 1989. Interleu- kin 1 and tumor necrosis factor stimulate human vascular endothelial cells to promote transendothelial neutrophil passage. J. Clin. Invest. 83:444-455.

48. Furie, M. B., and D. D. McHugh. 1989. Migration of neutrophils across endothelial monolayers is stimulated by treatment of the monolayers with interleukin-1 or tumor necrosis factor-alpha. J. Immunol. 143:3309-3317.

49. Huber, A. R., S. L. Kunkel, R. F. Todd III, and S. J. Weiss. 1991. Regulation of transendothelial neutrophil migration by endogenous interleukin-8. Science (Wash. DC). 254:99-105.

50. Anderson, D. C., R. Rothlein, S. D. Marlin, S. S. Krater, and C. W. Smith 1990. Impaired transendothelial migration by neonatal neutrophils: abnormalities of Mac-1 (CD1 lb/CD18)-dependent adherence reactions. Blood. 78:26132621.

51. Staunton, D. E., M. L. Dustin, H. P. Erickson, and T. A. Springer. 1990. The arrangement of the immunoglobulin-like domains of ICAM-1 and the binding sites for LFA-1 and rhinovirus. Cell. 61:243-254.

52. Dustin, M. L., R. Rothlein, A. K. Bhan, C. A. Dinarello, and T. A. Springer. 1986. Induction by IL-1 and interferon-gamma: tissue distribution, biochemistry, and function of a natural adherence molecule (ICAM-1). J. Im munol. 137:245-254. 